Review Article

\title{
EXOSOME NANOCARRIERS: BASIC BIOLOGY, DIAGNOSIS, NOVEL AND PERSPECTIVE APPROACH IN DRUG DELIVERY SYSTEMS: A REVIEW
}

\author{
P. V. KAMALA KUMARI, KETHA SRILEKHYA*, K. BHAVYA SINDHU, Y. SRINIVASA RAO \\ Department of Pharmaceutics, Vignan Institute of Pharmaceutical Technology, Duvvada, Visakhapatnam \\ *Email: kamalaparavastu@gmail.com
}

Received: 20 Jan 2021, Revised and Accepted: 14 Apr 2021

\begin{abstract}
Exosomes are the extracellular vesicles surrounded by a phospholipid bilayer shed from all cell varieties and plays a significant role in the communication and Transportation of materials between the cells due to their ability to transfer the proteins and nucleic acids from One cell to the another cell. Analogous in size and performance to synthetic nanoparticles, exosomes provide several Advantages, rendering them the foremost promising candidates for targeted drug or gene delivery vehicles. This review highlights the isolation techniques and delivery potential of exosomes, and equally presents research or analysis gaps for enhancing the use of natural vesicles for delivery functions. Exosome-based drug formulations can be applied to an extensive variety of diseases such as various infectious, cardiovascular, cancer and neurodegenerative disorders. Mostly, exosomes combine the benefits of both synthetic nanocarriers and cell-mediated drug delivery systems however avoiding their limitations
\end{abstract}

Keywords: Drug delivery, Exosomes, Functional therapeutics, Nanotechnology

(C) 2021 The Authors. Published by Innovare Academic Sciences Pvt Ltd. This is an open access article under the CC BY license (https://creativecommons.org/licenses/by/4.0/) DOI: https://dx.doi.org/10.22159/ijap.2021v13i4.40842. Journal homepage: https://innovareacademics.in/journals/index.php/ijap

\section{INTRODUCTION}

The term "exosome" was coined by Rose Johnstone in late 1970s, because the vesicles she found emerging from sheep reticulocytes structurally resembled endosomes, these particular ones were exiting material rather than entering [1]. Up to 1996 exosomes are regarded as the best waste-disposal vessels of cell homeostasis. This changed with the discovery of the B cells that can actually release functional antigentransferring exosomes. Hereafter the exosome scope has widened, over time attributing an array of the functionalities to these small extracellular entities. Cells can release multiple types of membrane vesicles, like plasma-membrane-budded microvesicles, multivesicular body-derived exosomes; Golgi apparatus-derived secretory vesicles or apoptotic bodies. All of these cell-derived microvesicles can carry biologically active cargo like proteins, mRNA and miRNA [2].

\section{Structure of exosomes}

Exosomes are a class of round-shaped lipid bilayer vesicles with a diameter ranging from $30-150 \mathrm{~nm}$, and in the image of exosomes $\mathrm{EM}$, we can see that their shape is round-shaped and the distribution is monodisperse [3]. They are known to be associated with diverse pathological conditions and represent the variety of functions like delivering biomolecules and genetic material to recipient cells.

Exosomes are enriched with the bundle of specific molecules which are different from proteins located in the plasma membrane including tetraspanins, heat-shock proteins, major histocompatibility molecules, cytoskeleton proteins (actin, tubulin, cofilin, myosin, vimentin, fibronectin, advillin, talin, CAP1, meosin, and keratin), microRNA (miRNA), messenger RNA (mRNA), Annexins (I, II, IV, V, VI, VII, and X1), apoptotic bodies [4].

Exosomes also contain a domain of lipids including sphingomyelin, ganglioside, ceramide and cholesterol called membrane rafts. There is an additional associated class of cytosolic proteins found in the exosomes includes Rabs, a family of GTPases involved in the vesicular transport and protein complexes that regulate the membrane fusion and docking. About $40 \mathrm{Rab}$ proteins have been identified in the exosomes RAB27A, RAB27B, RAB11, and RAB35 [5, 6]. Exosomes are special in their own way as they possess particular cell surface markers as a legacy from the cell of their origin. These cell surface markers provide exosomes the means of interaction with neighboring and distant cells [7, 8]. Possession of these potentials has made exosomes a very valuable therapeutic tool with inherent qualities in the biomedicine research area for targeted drug delivery to counter deadly diseases. In addition to proteins, lipids and mRNA, exosomes also transfer miRNA to recipient cells which can alter the pathologic genetic expression.

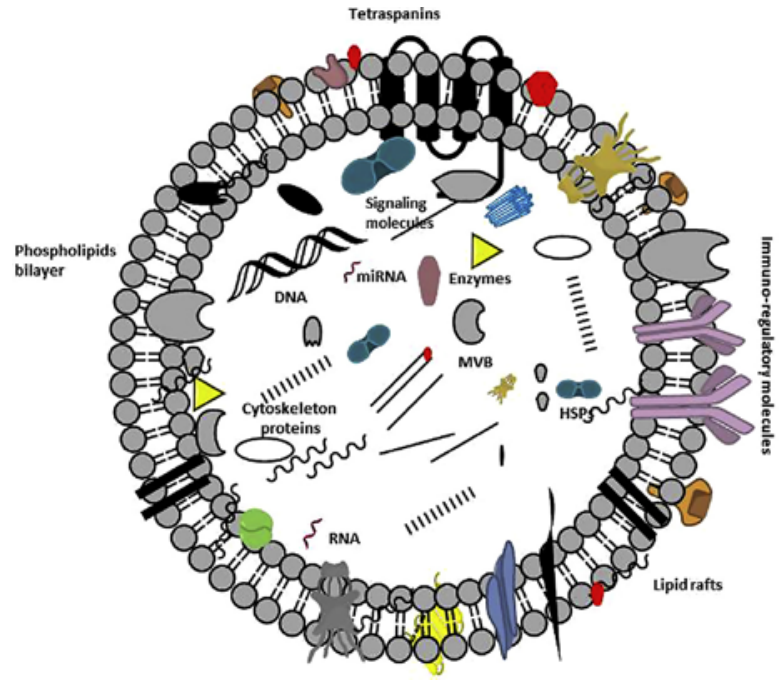

Fig. 1: Structural components of the exosome. Exosomes are composed of a bundle of specific molecules including tetraspanins (CD9, CD63, CD81, CD82), HSPs (HSP60, HSP70, HSPA5, CCT2, and HSP90), MHCs (MHC-I and-II), cytoskeleton proteins (actin, tubulin, cofilin, myosin, vimentin, fibronectin advillin, talin, CAP1, keratins, meosin), micro RNA and mRNA

Annexins I, II, IV, V, VI, VII and X1, and apoptotic bodies [8]

Biogenesis, release, and uptake of exosomes

The secretion of exosomes in the cells occurs by fusion of the Mult Vesicular Bodies (MVB'S) and the cell membranes most vibrant compartments which are actively involved in the internalization of extracellular ligands, post membranous invagination, and the release of different types of endocytic vesicles. Exosomes are formed by the inward budding of the endocytic vesicles and also contain cytoplasmic components. Continuous formation of the exosomes leads to the development of the MVBs. The membrane of MVBs is semipermeable and ensures that cargoes are selectively loaded into 
these exosomes. Exosomes can have similar kinds of membrane constituents even if they have originated from different types of cells [9]. The preliminary steps involved in sorting out the proteins undergoing endocytosis are the inward budding processes on the cell surface, which can be the clathrin-dependent and independent ways. The endocytotic vesicles are then transferred to endosomes which have an internal acidic environment. The acidic $\mathrm{pH}$ gets reduced by some modification of protein composition in the late endosomes which gets shed into the endosomal lumen to form MVBs and further leads to the formation of the Intra Alluminal Vesicles (ILVs). MVBs are abundant in cholesterol which can have dire consequences for cell survival, so they get fused with the plasma membrane and release exosomes and MVBs with a low amount of cholesterol fuse with the lysosomes and are involved in the proteolysis. There are mainly two kinds of mechanisms involved in the formation of exosomes; ESCRT (endosomal sorting complexes required for transport) dependent (which comprises four proteins 0 , I, II, III, and lipid-dependent) and ESCRT independent. ESCRT-O separates ubiquitinated proteins, I and II complexes help in the budding, and III completes the process and leads to the formation of ILVs in the MVBs rich population.

Exosomes primarily originate as ILVs then gets matured to MVEs $[10,11]$. Tetraspanins such as the CD81, CD9, and CD63 play an important role in the composition of ESCRT-independent loading into the exosomes by organizing the membrane into TetraspaninEnriched domains. The ESCRT complex mechanism, which is both dependent and independent, for packing of the protein cargo into exosomes is a sign of the existence of different MVB and exosome populations. Different types of cancerous cells secrete various types of exosomes which may vary in the morphology and miRNA composition [12, 13], but some secrete exosomes which get differentiated by these surface antigens having different protein content with various functions. The exosomes released from the apical and basolateral cell membranes may have the heterogeneous population of vesicles, but the existence of the controlled and specialized mechanisms control the selective, specific sorting of the protein and miRNA cargo into these vesicles. Numerous researchers explained about the biogenesis of exosomes, most of these mechanisms are still unclear.

\section{Isolation and purification of exosomes}

\section{Isolation by differential ultracentrifugation}

This method has become the gold standard process for the isolation and purification of the exosomes and exosome-like particles mainly due to its simplicity and cost-effectiveness [1418]. This method relies on differences in density and size of the separated particles. The exosome source, mainly the biological fluids or plant juice, are subjected to the series of centrifugation process with increasing speeds and duration to eliminate the larger and higher-density components; the pellets which are derived from this step are discarded. The final round of centrifugation of the supernatant is carried out at a higher speed of about $100,000 \times \mathrm{g}$ to recover the pellet containing the exosomes, which is resuspended and washed in a buffer. This method is often modified for the different sources of the exosomes. For instance, for animal fluids (e. g., plasma), due to their viscosity, dilution of the fluid and centrifugation at increased speed and duration are necessary for obtaining high exosome yields. Differential ultra-centrifugation is the general process for exosome isolation and the resulting exosomes often contain contaminants, mainly nucleic acids and protein aggregates [19]. For further purification, an extra step can be added involving 30\% sucrose gradient. Exosomes float on a sucrose gradient hence, this step is invaluable for obtaining pure exosomes. This process ensures that the majority of the contaminants isolated with the exosomes does not float with the exosomes and, thus, would be separated [20].

\section{Isolation by ultrafiltration}

Due to the low purity of the exosomes isolated by differential ultracentrifugation, efforts have been made to devise methods for large-scale isolation of exosomes with both higher purity and yield. Ultrafiltration is a technique that separates biomolecules based on different sizes Lobb group [21] suggested this process as a faster alternative to ultracentrifugation when they observed that it led to a higher recovery of exosome particles. This method involves the use of membrane filters and pressure to eliminate large molecular size contaminants with subsequent isolation of exosomes. An emerging modification to this procedure involves the separation of the ultrafiltration product by liquid chromatography for further purification $[22,23]$. This extra step is expected to increase the production cost, which is considered important when using exosomes in food applications.

\section{Isolation by immuno isolation}

In this process, antibodies coat the magnetic beads that target the proteins present on the exosome surface. This method relies on surface biomarkers that are differentially expressed on exosomes [14]. Although it ensures the isolation with higher purity, the major drawback of this process is the limited information available on the exosomal structures and surface compositions, especially for new plant sources. Besides, the process would not be efficient for largescale isolation of exosomes due to the dissociation and purification steps essential after their binding to the antibodies.

Table 1: Identification and quantification methods

\begin{tabular}{|c|c|c|c|c|}
\hline Methods & Principle & Advantages & Disadvantages & References \\
\hline $\begin{array}{l}\text { Dynamic } \\
\text { Light } \\
\text { Scattering }\end{array}$ & $\begin{array}{l}\text { Sample is exposed to a monochromatic light and an } \\
\text { appropriate detector detects the signal. To study } \\
\text { the scattering of light from absorbing and non- } \\
\text { absorbing particles that are large compared to the } \\
\text { wavelength of light by taking into consideration } \\
\text { particle shape and the difference in refractive index } \\
\text { between particles and the medium the particles are } \\
\text { present in. }\end{array}$ & $\begin{array}{l}\text { 1. Measurement of } \\
\text { molecules with } M W<1000 \\
\text { Da. } \\
\text { 2. require low volume i.e. } 2 \\
\text { } 2 \text {. }\end{array}$ & $\begin{array}{l}\text { DLS measurements are very } \\
\text { sensitive to temperature and } \\
\text { solvent viscosity. Hence the } \\
\text { temperature must be kept } \\
\text { constant and solvent } \\
\text { viscosity must be known for a } \\
\text { reliable DLS experiment. }\end{array}$ & [15] \\
\hline $\begin{array}{l}\text { Nanoparticle } \\
\text { Tracking } \\
\text { Analysis }\end{array}$ & $\begin{array}{l}\text { It is a ubiquitous requirement in a broad range of } \\
\text { industry sectors. Product performance and stability } \\
\text { depends on the ability to manufacture particle } \\
\text { suspensions to fine tolerances without the presence } \\
\text { of contaminants or aggregates. }\end{array}$ & $\begin{array}{l}\text { 1. Single vesicles detection } \\
\text { with a diameter of } 50 \mathrm{~nm} \text {. }\end{array}$ & $\begin{array}{l}\text { Exosomes preparation is only } \\
\text { semi-quantification and } \\
\text { considerable intra-assay } \\
\text { count variability exists. }\end{array}$ & {$[16]$} \\
\hline $\begin{array}{l}\text { Surface } \\
\text { Plasmon } \\
\text { Resonance }\end{array}$ & $\begin{array}{l}\text { The energy of photons can be used for many useful } \\
\text { chemical reactions, including solar production of } \\
\text { fuels and water purification. While these } \\
\text { photocatalytic processes are potentially very useful } \\
\text { and their efficiency is far too low for practical large } \\
\text { scale applications. }\end{array}$ & $\begin{array}{l}1 . \quad \text { Require small } \\
\text { sample volumes and } \\
\text { competence of total mass } \\
\text { of exosomes, including } \\
\text { proteins, lipids and } \\
\text { nucleotides. }\end{array}$ & $\begin{array}{l}\text { Quality control is inadequate } \\
\text { and normalization across } \\
\text { study groups. }\end{array}$ & [17] \\
\hline
\end{tabular}




\section{Exosome characterization}

The characterization of exosomes can be summarized by its size, protein and lipid content. Generally, they can be identified by detecting the morphology, particle size, and surface markers on the surface of exosomes. Methods like transmission electron microscopy (TEM), scanning electron microscopy (SEM), cryo-electron microscopy and atomic force microscopy (AFM) are commonly employed to characterize the microstructure of exosomes. Nanoparticle tracking analysis (NTA) and dynamic light scattering (DLS) are two attractive techniques for measuring the distribution of particles in suspension. NTA is an optical particle tracking technique developed rapidly to realtime monitor the size distribution and concentration of particles. The concentrations of exosomes and vesicles were determined from biological fluids by surface plasmon resonance (SPR). Some of the identification methods were summarised in table 1.

\section{Preparation of exosomes and exosome-mimetics}

\section{Drug loading methodologies}

The drug loading methodologies applied to date in the case of exosomes, and their physical mimetics (Cellular vesicles), are categorized into two main groups, the pre-loading methods and the post-loading methods.

In pre-loading methods, the drug is initially produced or loaded in the parental cells, and therefore, the extracellular vesicles or cellular vesicles isolated or produced by them are previously pre-loaded with the desired drug. Such methodologies are predominantly useful when oligonucleotides or proteins are to be loaded in the vesicles, in which case the cells can be programmed to produce "a-la-carte" extracellular vesicles or cellular vesicles.

In post-loading methods, the drug is loaded in the extracellular vesicles after their separation. Though, we have not been able to notice any reports regarding the potential to use superior loading methods that have been very successful for effective loading of amphiphilic drugs into liposomes, such as the "active or remote" loading approaches for amphiphilic drugs, such as Doxorubicin etc, which can be established as a "one-stop" manufacturing methods on a microfluidic setup, as recently proven $[24,25]$.

\section{Preloading methods}

\section{Treatment of parental cells with drugs}

In this technique, the parental cells are treated with a drug, and the cells then secrete drug-pre-loaded exosomes or extracellular vesicles. Although it is impossible to regulate the loading efficiency with this type of methods, several studies, which are summarized below, have been conducted using such techniques, owing to their simplicity. Encapsulated paclitaxel (PTX) into murine SR4987 mesenchymal stroma cells after culturing the cells with a low dose of PTX for $24 \mathrm{~h}$, and then, washing and reseeding them with fresh medium. After $48 \mathrm{~h}$, PTX-loaded-exosomes primed with high dose of PTX were collected. The PTX-loaded exosomes presented strong anti-proliferative activity against CFPAC-1 human pancreatic cells, as associated with control exosomes which were isolated from untreated cells [21].

Isolated extracellular vesicles from resistant hepatocellular carcinoma cells (HepG2) cells treated with different drugs (carboplatin paclitaxel, etoposide and irinotecan hydrochloride) were isolated. After exposure to the anticancer drugs, membrane microvesicles established by TEM and Western Blot to be exosomes were actively released by the cells. The later exosomes differed in their capability to present heat shock proteins (HSPs) on the cell surface. Their size ranged between 30 and $100 \mathrm{~nm}$ with the bulk of the vesicles being around 70-90 nm [22]. Synthetic fusogenic liposomes which were loaded with hydrophobic drugs were efficiently incorporated into the host cell membrane and the drugs were subsequently loaded into the membranes of the cell-secreted extracellular vesicles [27]. The same group used this method to introduce lipid-azides in the membrane of extracellular vesicles which were extra conjugated with targeting moieties and loaded with Paclitaxel (PTX) and tirapazamine (TPZ) [28].

\section{Parental cell engineering}

In the last decade, an increasing number of reports in the literature define methods for successful loading of therapeutic cargo in exosomes, by modulation of the exosome-producing parental cells. The most common cell engineering techniques include transfection or activation of parental cells.

Transfection is the most widely used and effective method for the loading of therapeutic proteins or oligonucleotides into exosomes. RNA (miRNA, siRNA, and mRNA) and protein sequences are simply transfected as synthetic oligonucleotides or expressed from a plasmid backbone. Cell transfections are carried out by the calcium phosphate method or by commercially available lipid transfection components such as lipofectamine, or others. With such approaches, biological cargos can be packaged into exosomes to promote or silence gene expression or regulate transcription in recipient cells. Studies have revealed that transfection can also be used to overexpress a specific protein on the surface membrane of the exosomes, or the proteins could be packaged into the exosome lumen. Certainly, in addition to modifying exosome membranes through genetic engineering of their parent cell, the therapeutic cargo of exosomes may also be manipulated by altering various aspects of their regulated biogenesis.

miRNAs were effectively introduced into exosomes in several studies by using miRNA expression vectors. Transfecting modified miR-143 in THP-1 macrophage cells led to the successful loading of the modified miRNA into the exosomes are presented and this work showed that when overexpressing a miRNA into parental cells, this effectively leads to the passive load of miRNA into exosomes [24]. The miRNA-loaded exosomes can also be targeted efficiently to recipient cells after engineering their surface with targeting peptides, and proven that intravenously injected exosomes accumulated in the tumor site and reduced the tumor growth [25].

Interestingly, cell activation has been used as a method for loading functional cargo into exosomes. Though it is not the most suitable method for exosome loading, it shed light on some characteristics of the physiology and function of exosomes. Cell activation as a methodology for exosome loading was, though, only studied a few times.

\section{Post-loading methods}

In this section, the post-loading methods which have been used to date for drug loading into engineered exosomes of artificialexosomes are defined, starting from the simplest methods and continuing with the most complex ones (usually applied in liposome technology).

\section{Incubation with drug}

The simplest way to incorporate any cargo into exosomes is their coincubation, merely by mixing the isolated vesicles with the drug. The driving force for the loading is the altered concentration of the drug in and out of the vesicle membrane. The low loading capacity is considered as the main disadvantage of this method. The loading efficiency of co-incubation process depends on the lipophilic properties of the drug, as well as the concentration gradient [32]. Certain examples of drug loading into exosomes by their incubation with the drug of interest are given below:

Incubation of curcumin with exosomes in PBS at $22^{\circ} \mathrm{C}$ for 5 min and the exosomes were later purified [26]. Curcumin was hence selfassembled into the lipid bilayer of exosomes over hydroscopic interactions, resulting in improved drug stability. Using the similar method, Encapsulation both curcumin (Exo-Cur) and a signal transducer and activator of transcription 3 (Stat3) inhibitor (ExoJSI124), by mixing curcumin or JSI124 with EL-4 cell-derived exosomes in PBS. Drug-loaded exosomes (Exo-cur or Exo-JSI124) were subsequently separated after incubating at $22{ }^{\circ} \mathrm{C}$ for $5 \mathrm{~min}$. Recognition of an efficient, robust and highly-reproducible technique for loading therapeutic RNA into exosomes upon coincubation of hydrophobically altered small interfering RNAs (hsiRNAs) and exosomes, without affecting both the vesicle size and their integrity [27]. Loading of porphyrins of different hydrophobicities into exosomes by incubation with the drug at RT 
for 10 min and effective loading of the enzyme catalase (a tetramer protein of $250 \mathrm{kDa}$ ) into RAW264.7 cell-extracted exosomes in PBS at RT for $18 \mathrm{~h}$ [28].

\section{Electroporation}

By this technique, an electrical field distracts the phospholipid bilayer of vesicles (such as extracellular vesicles or exosomes or cells), making small pores in their membrane, and therefore allowing the passage of the drug into the vesicles. The integrity of the vesicle membrane is then recovered, causing the development of drug-loaded vesicles [30].

Electroporation was performed at $200 \Omega, 500 \mu \mathrm{F}, 200 \mathrm{mV}$ and a pulse time of $20-30 \mathrm{~ms}$ [35]. Further examples of drug loading into exosomes by electroporation include DOX loading into mouse immature dendritic cells (engineered to express Lamp2b (an exosomal membrane protein) fused to $\alpha v$ integrin-specific iRGD peptide (CRGDKGPDC)) derived exosomes. The purified exosomes were capably loaded with DOX via electroporation.

\section{Sonication}

In this process, exosomes derived from donor cells are mixed with drugs and subsequently sonicated by a probe sonicator which allows the drug to flow into the exosomes due to the sonication-induced deformation of their membrane. In principle, the reformation of exosomal membranes under sonication may allow the passage of drugs across relatively tight lipid bilayers [37]. Specific examples of drug loading with sonication are presented below:

Sonication is a method to load paclitaxel (PTX) into macrophagereleased exosomes; the method resulted in great loading efficiency and sustained drug release, while it did not significantly affect the protein or the lipid contents of the exosomes [31]. Sonication was also used as a process to load small RNAs (siRNA and miRNA) into exosomes (and microvesicles). However, the application of exosomes for therapeutic RNA delivery may be inadequate by such loading approaches that may induce cargo aggregation or degradation. Functional small RNAs (siRNA, miRNA or single-stranded DNA (ssDNA)) into exosomes isolated from two altered cell lines were loaded, HEK293T and MCF-7 by sonication. For this, the nucleic acids were incubated with the exosomes at RT for 30 min and sonicated in a bath sonicator at $35 \mathrm{kHz}$ for $30 \mathrm{~s}$. Then the Mixtures were placed on ice for $1 \mathrm{~min}$ and sonicated again for the same time period. These conditions led to minimal detectable aggregation probably because of the minor sonication conditions applied [32].

\section{Extrusion}

In this process, exosomes are mixed with a drug, and the mixture is loaded into a syringe-based lipid extruder and extruded through membranes with $100-400 \mathrm{~nm}$ porous size, at controlled temperature. During the extrusion, the exosome membrane is interrupted and vigorously mixed with the drug, resulting in drug loading into the exosomes.

Loaded porphyrins of altered hydrophobicities into exosomes by extrusion were loaded. The procedure was carried out at $42{ }^{\circ} \mathrm{C}$ using a syringe-based, hand-held mini-extruder equipped with a heating block, and polycarbonate membranes of $400 \mathrm{~nm}$ pore size. Each sample was extruded 31 times. It was found that the extrusion method caused in alteration of the zeta potential of the vesicles, due to modulation of the constitution of the lipid membrane of original exosomes. The extruded exosomes were demonstrated to cause cytotoxicity, whereas exosomes loaded with similar porphyrin but prepared by other methods did not show significant cytotoxicity. Catalase has similarly been loaded into RAW264.7 macrophage-derived exosomes by extrusion. The catalaseexosome mixture was efficiently extruded ( $\times 10$ times) through $200 \mathrm{~nm}$ pore diameter filters [28].

\section{Freeze/thaw cycle method}

In this process, drugs are mixed and incubated with exosomes at RT, and the mixture is then frozen at $-80{ }^{\circ} \mathrm{C}$ or in liquid nitrogen, and rethawed at RT. This process is repeated for at least 3 cycles to safeguard drug encapsulation [42]. However, by this method exosomes, may aggregate, while the drug loading efficiency is usually lower than that of sonication or extrusion.
The freeze-thaw technique has been used for catalase incorporation into exosomes. The catalase solution was mixed with the exosomes, freezed at $-80{ }^{\circ} \mathrm{C}$, incubated for $30 \mathrm{~min}$ and thawed at RT. The freeze-thaw cycle was repeated for three times [36].

A new approach to formulate engineered hybrid exosomes by fusing the membranes of exosomes with those of liposomes, using a freezethaw method for this is proposed and Raw 264.7 cell-derived exosomes were mixed with fluorescently-labeled liposomes composed of phospholipids, pegylated-phospholipids, and fluorescently labelled phospholipids. These mixtures were frozen in liquid nitrogen and thawed at RT for $15 \mathrm{~min}$. To examine the applicability of the membrane fusion technique, they investigated the fusion behavior between exosomes bearing a specific membrane protein (HER2) and liposomes. HER2 and phosphorylated HER2 were detected in the exosome-liposome mixtures, representing that exosome-liposome hybrids carrying specific proteins can be obtained by freeze-thaw methods. Cellular uptake studies performed using the hybrid exosomes shown that the interactions between the developed exosomes and cells can be changed by varying the lipid composition, and consequently, also the properties of liposomes [33].

\section{Saponin-assisted loading}

Saponin is named from the Latin "sapo" which means "soap"; it is a surfactant molecule that, upon incubation with exosomes, produces pores in their membrane through interactions with cholesterol, leading to increased exosomes-membrane permeability. The sugar chain can contain from one to numerous monosaccharide residues, and is usually attached at C-3. The surface activity, as well as some additional biological functions of saponins (including their haemolytic activity), are attributed to their characteristic structural features and their amphiphilic nature which is are the results of the presence of a hydrophilic sugar moiety and a hydrophobic genin (called sapogenin) [34].

Catalase into exosomes ex vivo using different methods were loaded by the saponin-assisted process, a mixture of catalase and exosomes was supplemented with $0.2 \%$ saponin and placed on a shaker for 20 min at RT. This technique caused in high loading efficiency sustained release, and catalase preservation against proteases degradation. Although saponin is a surface-active agent, it did not degrade catalase, whose activity was preserved [29].

Saponin can also help in loading other hydrophilic molecules into exosomes. For saponin-assisted drug loading of hydrophilic porphyrins, exosomes and the drug were incubated with $0.1 \mathrm{mg} / \mathrm{ml}$ saponin at RT for $10 \mathrm{~min}$ and high drug loading was assessed by this method [35].

Though, there are concerns regarding the in vivo haemolytic activity of saponin. Haemolysis of red blood cells seems to result from the capability of saponin to form complexes with the cholesterol of the cell membrane, leading to the development of pores, cell membrane permeabilization, and alterations in the negatively-charged carbohydrate portions on the cell surface. In any circumstance, when used as a method to assist drug-loading into exosomes, the concentration of saponin should be kept to a minimum, and the exosomes should be washed instantly after incubation with saponin [39].

\section{Applications of exosomes in the pathogenesis, diagnostics, and therapeutics}

Exosomes show a wide variety of functions in the cell including cell communication, diagnostic markers, and potential roles in therapeutics. Modern studies demonstrated the possible role of exosomes as a biomarker for early diagnosis of diseases [40, 41]. In the past few years more attention has been focused on the role of exosomes in the pathophysiology of a variety of diseases including metabolic disorders, cancer, cardiovascular diseases, immune diseases, and neurodegenerative diseases.

\section{Exosomes in inflammatory diseases}

Exosomes are known to play a vital role in treating a variety of autoimmune diseases and inflammatory reactions as potential therapeutic agents. On the other hand, exosomes released from infected cells demonstrate a variety of functions. Further, the 
exosomes released from pathogen-stimulated brain cells have miRNAs which could interfere with the gene expression of different other cells [42]. In asthmatic patients, the release of exosomes from the bronchial epithelial cells in the lungs is enhanced and might be responsible for the enhancement in intercellular signalling. Exosomes those are released from the epithelial cells which are treated with the interleukins 13 (IL-13) stimulate the inflammation along with cell proliferation, but some specific drugs that result in the inhibition of exosomes production can lead to diminishing the inflammation [43].

Exosomes from Mycobacterium tuberculosis-infected macrophages contain mycobacteria-derived antigens and cause the induction of inflammatory immune responses while inhibiting IFN $-\gamma$ dependent activation of the macrophages $[44,45]$. In the same way, exosomes released from Mycobacterium avium or Mycobacterium smegmatis infected macrophages have great Hsp70 expression; though, the stimulation of the nuclear factor kappa-light-chain-enhancer of activated B-cells (NF-kB) pathway helps producing high levels of the tumor necrosis factor- $\alpha$ (TNF- $\alpha)[37,38]$. Exosomes with Hsp70 expression could produce a pro-inflammatory response with the release of TNF- $\kappa$ B by macrophages and promotion of NK cell activity [46]. The Curcumin-loaded exosomes are used to activate myeloid cells, which are involved in the control and development of autoimmune diseases associated with inflammation (EL-4) [32]. Exosomal curcumin helps in the decrease of the number of CD11b1Gr-11 cells which are responsible for causing acute lung inflammation by increasing the solubility, stability, and bioavailability of curcumin after encapsulation into the lipid bilayer of the exosomes. Nevertheless, it becomes easier to deliver the encapsulated curcumin to the brain via intranasal administration. Earlier studies have exhibited that exosomes those are secreted from cancerous cells release certain cytokines into the microenvironment which are recognised with triggering of the immune response against tumorigenic tissue [47, 48]. On the contrary, exosomes that are derived from tumor tissue demonstrate exactly the opposite immune response in a manner of bearing different immunosuppressive molecules. This happens either because of decline in CD41, CD81, and natural killer cell stimulation or amplification in the differentiation of the immunosuppressive cells [5]. Injecting of exosomes obtained from tumor cells helps in supporting metastasis by decreasing natural killer cells activation, but increased the differentiation of myeloid cells and lead to the development of melanoma and carcinoma [49]. Till date, there are no relevant explanations given for these controversial results by different scientists, but it could be speculated to be due to the heterogeneous population of exosomes.

\section{Exosomes in cancer}

Cancer is the foremost cause of mortality worldwide. Existing treatment methods include surgery, chemotherapy, and radiation which further raises toxicity concerns. Since these methods are unable to cure metastasis of the cancerous cells there is an excessive need to develop more effective and less toxic therapies. Immunotherapy is a much-explored way of treating many types of cancers. Antibodies have presented a clinical accomplishment for cancer immunotherapy as well [38]. Latest research reports show the potential role of exosomes in cancer treatment as well as diagnosis and prognosis [50-55]. The RNA and proteins content from tumor-derived exosomes are involved in disseminating malignancy to recipient cells. This provides diagnostic information and aid in therapeutic selection for cancer patients. The most significant fact about exosomes is their ability to stimulate the immune response that can be exploited for the development of cancer vaccines [56].

\section{Exosomes in neurodegenerative diseases}

Exosomes are involved in a variety of neurological disorders including Alzheimer's disease (AD) and Parkinson's disease (PD). They are released by numerous cell types like normal or pathogenic, into the extracellular environment. Mostly, exosomes released by tumor tissues initiate the metastasis and highlight the fact that exosomes have the ability to spread diseases within the body [57]. This ability of exosomes to spread the diseases is measured to be responsible for neurodegenerative disorders. Although the accurate mechanism of transmission is yet to be discovered, numerous studies have revealed that it could be either by cell to cell communication or via tunneling nanotubes $[58,59]$. Various research studies have demonstrated the involvement of exosomes in the transmission as neurodegenerative proteins were found to be secreted from the cell in association with exosomes. Due to their small size and relative stability, exosomes can cross the blood-brain barrier (BBB) therefore, reveal bright perspectives toward diagnosis and as therapeutic tools for future neuro medicine [58].

\section{Exosomes as nanocarriers}

Nanoparticle drug delivery systems deliver many benefits like their ability to load high quantities ability to load both drug along with the adjuvant for the introduction of costimulation within a specific nanocarrier, sustained release of the loaded drug. Different types of nanocarriers have been considered at length for the targeted drug delivery of drugs or genes to a specific site of cancerous tissue. Nanocarriers are most-exploited in this field because they are more permeable along with the ability to accumulate into tumors passively as well as having the competence of longer retention, for example, DCs. Macrophages and primary cells are the natural phagocytotic involved in antitumor immunity and inflammatory response hence, Nano-drug delivery systems are the promising platforms in delivering immunomodulatory compounds to these cell. Exosomes, also called biological nanocarriers, are becoming visible as a promising alternative to this new theory of drug delivery [59].

\section{Drug delivery vehicles for nucleic acids}

Exosomes are exceptionally outstanding delivery systems which can also carry DNA and RNA-like big and heavy molecules to the target cells, thus instigating genetic modification in the biological processes. This approach can be employed as a promising tool for delivering genetic material and altering the gene expression in certain diseases and help progress gene therapy.

\section{Delivery application of exosomes}

Exosomes expanded a lot of interest over the past decade because of their potential as a Nano delivery system, and advantages over other delivery systems. For instance, exosome vesicles form innately and naturally contribute as nanocarriers. Exosomes also have a target ability benefit as they bind specific targets due to their unique surface compositions. On reaching their destination, exosomes can discharge their contents into the target cells through either phagocytosis or membrane fusion [60]. Although exosomes generally have a natural targeting capability, which differs depending on their source, their preferred target can be customized by inserting molecules in donor cells (sources) that would be recognized by the recipient cells in the body. This method includes inserting genes that code for proteins that target specific cells so that exosomes secreted from those cells possess the desired targeting capability.

\section{Mammalian exosomes as a delivery}

Vehicle for nutraceutical compounds in planning a drug delivery system for Parkinson's disease [36], specified the efficient encapsulation and stabilization of catalase with exosomes isolated from murine monocytes and macrophages. A size range of 100-200 $\mathrm{nm}$ was obtained for the catalase-loaded exosomes, which had a high loading efficiency, sustained release, its content preserved from degradation by proteases, and important in vitro and in vivo neuroprotective properties. In one study, exosome encapsulation enriched the bioavailability and anti-inflammatory activity of curcumin and ensured target specificity toward inflammatory cells [32]. The attention providing to animal-derived exosomes as delivery vehicles for bioactive compounds is because of their biocompatibility, biorecognition, target specificity, and stability.

\section{Delivery of bioactive plant components}

Exosome-like particles derived from plants have recently extended a lot of interest. The vesicles, situated in the paramural space of plants, are associated in structure and function to their mammalian counterparts and are recognised as plant-derived edible nanoparticles (PDENs). Majority of the plant sources have characteristic and unique compounds with physiologically relevant 
bioactivities. It is probable that the bioactive compounds are also delivered to their targets via exosome-like vesicles. It was reported that ginger-derived exosomes possess high levels of phosphatidic acid, which promotes intravesicular fusion for target delivery; there would be a necessity to offload the inherent bioactive compounds in exosomes in order to reach a great loading efficiency with the bioactive compounds of interest.

\section{Research studies of exosomes as drug delivery systems}

Exosomes are $30-100 \mathrm{~nm}$ nanoparticles secreted by cells into the extracellular environment. The possibility of nanoparticle exosomes as a carrier to entrap curcumin, curcumin was mixed with exosomes and then subjected to sucrose gradient centrifugation Exosomal protein markers, such as TSG101 and CD81 were identified in the exosomal curcumin [61]. Recently, exosomes have been employed to deliver exogenous nucleic acid drugs to specific cell types or tissues in vivo especially tumor tissues and they have shown great promise as naturally occurring nano-sized drug carriers. First example of using exosomes is for the targeted delivery of the chemotherapeutic drug Doxorubicin to solid tumors. Systemic administration of Doxorubicin delivery system significantly inhibited tumor growth causing no over toxicity. Studies suggest that exosomes appear to be superior candidates for specifically transporting therapeutic drugs to tumors resulting in significant inhibition of tumor growth [62]. Fluorescent doxorubicin and paclitaxel alone are formulated with bEND3 exosomes were prepared and penetration into brain tissue was evaluated. When given alone, the images showed no distribution of fluorescent drug out of the vasculature and into the brain. However, when formulated with exosomes both drugs are distributed into the brain region. These results indicate that exosomes can effectively deliver the anticancer drugs across the BBB into the brain in vivo [63]. Exogenous siRNAs were successfully introduced into various kinds of human exosomes and were used to deliver siRNA to human mononuclear blood cells. Plasma exosomes effectively delivered the siRNA into the target cells $[66,67]$. Some of the research studies conducted using exosomes as drug delivery systems were summarized in table 2 .

Table 2: Summary of studies conducted using exosomes as drug delivery systems

\begin{tabular}{lll}
\hline Research & Aim & References \\
\hline Small molecules & Using exosomes to improve the delivery of curcumin & {$[61]$} \\
& Delivery of doxorubicin into tumor tissue & {$[62]$} \\
& Deliver paclitaxel and doxorubicin across blood-brain barrier & {$[63]$} \\
Protein & Deliver catalase across BBB to treat PD & {$[64]$} \\
siRNA & Human exosomes to deliver siRNA into T cells & {$[65]$} \\
& Exosomes to deliver siRNA into HeLa and Fibrosarcoma cell line (HT1080 cells) & {$[66]$} \\
miRNA & Delivery of siRNA using endothelial-derived exosomes & {$[67]$} \\
\hline
\end{tabular}

\section{Advantages and disadvantages}

A proportion of drug compounds in the in vivo conditions are very unstable and have many challenges like drug resistance, low access to target tissue and so on for successful treatment. On the other hand, because of the problems associated with many drug delivery systems such as cytotoxicity of organic and non-organic nanoparticles, the improvement of delivery systems based on biological patterns is very important. Because of the negligible size of the exosomes between $30-150 \mathrm{~nm}$ along with their biocompatibility and hydrophilicity, and cheapness compared to nanomaterials, this vehicle can progress the constancy of drugs by loading the drug in the interior space and even on its outer surfaces through preventing macrophage phagocytosis. While exosomes also increase the cellular uptake in drug resistance by cancer cells. However, the lack of a complete understanding of the nature of exosomes due to their heterogeneity caused from undesirable separation and lack of purity in production, as well as the possibility of exacerbation of the disease due to the presence of exosomes that have the same activity as metastatic cancer exosomes, has been confronted with uncertainties and fear in clinical practices.

\section{Challenges of exosome-based nanocarriers for drug delivery}

The major drawback with the use of exosomes as a delivery agent is centered on the isolation process, inefficient extraction, low extraction yield, low encapsulation and loading efficiencies, and issues with delivery of exogenous hydrophilic macromolecules, and potential delivery of undesired cargo materials naturally present in the exosomes. Maximum exosome extraction methods are complex, laborintensive, and give low yield. However, recent extraction and detection techniques have shown promise, such as magnetic adhesion and flow cytometry in addition to better loading techniques such as sonication [38], electroporation and incubation. However, offloading the content of the exosome without distorting the structural integrity is very substantial in ensuring high loading and encapsulation efficiencies. Finally, there is a necessity for exosome functionalization to accommodate the encapsulation of exogenous hydrophilic macromolecules both by pre-formation (i.e., during exosome biogenesis) or post-formation loading (i.e., after exosome isolation).

\section{CONCLUSION}

Exosomes are nanosized vesicles secreted by a range of variety of cells and have ideal features as nanocarriers for drug and gene delivery.
Exosomes are well-known to be associated with diverse pathologies and represent a variety of functions like delivering biomolecules such as mRNAs, miRNAs, siRNA, drugs and proteins to the recipient cells by communication at intercellular and tissue levels. Exosomes show a wide variety of functions in the cell including, diagnostic markers, and their potential role in therapeutics. Certain exosomes comprise of immunostimulatory or immunosuppressive effects which can be used as immunotherapies for cancer or autoimmune diseases. Emerging evidence has discovered the promising therapeutic potential of exosomes as effective nano-vaccines and immunological treatment for inflammatory diseases. Further investigation is required to fully elucidate the use of exosomes in diagnostic and therapeutic measures.

\section{ACKNOWLEDGEMENT}

The authors are thankful to Dr L. Rathaiah, Chairman, Vignan Group of institutions for providing the necessary online facilities to complete the review.

\section{FUNDING}

Nil

\section{AUTHORS CONTRIBUTIONS}

All the authors have contributed equally.

\section{CONFLICT OF INTERESTS}

The authors declare no conflict of interest.

\section{REFERENCES}

1. Boorn JGV, Daßler J, Coch C, Schlee M, Hartmann G. Exosomes as nucleic acid nanocarriers. Adv Drug Delivery Rev 2013;65:331-5.

2. Raposo G, Nijman HW, Stoorvogel W. B lymphocytes secrete antigen-presenting vesicles. J Exp Med 1996;183:1161-72.

3. Record M, Carayon K, Poirot M, Silvente Poirot S. Exosomes as new vesicular lipid transporters involved in cell-cell communication and various pathophysiologies. Biochim Biophysica Acta Molecular Cell Biol Lipids 2014;1841:108-20.

4. Akers JC, Gonda D, Kim R, Carter BS, Chen CC. Biogenesis of extracellular vesicles (EV): exosomes, microvesicles, retrovirus-like vesicles, and apoptotic bodies. J Neuro-Oncol 2013;113:1-11.

5. Bobrie A, Colombo M, Raposo G, Théry C. Exosome secretion: molecular mechanisms and roles in immune responses. Traffic 2011;12:1659-68. 
6. Skokos D, Botros HG, Demeure C. Mast cell-derived exosomes induce phenotypic and functional maturation of dendritic cells and elicit specific immune responses in vivo. J Immunol 2003;170:3037-45

7. Bartel DP. MicroRNAs: genomics, biogenesis, mechanism, and function. Cell 2004;116:281-97.

8. Huotari J, Helenius A. Endosome maturation. EMBO J 2011;30:3481-500.

9. Hanson PI, Cashikar A. Multivesicular body morphogenesis. Annual Rev Cell Dev Biol 2012;28:337-62.

10. Palma J, Yaddanapudi SC, Pigati L. MicroRNAs are exported from malignant cells in customized particles. Nucleic Acids Res 2012;40:9125-38.

11. Penfornis P, Vallabhaneni KC, Whitt J, Pochampally R. Extracellular vesicles as carriers of microRNA, proteins and lipids in tumor microenvironment. Int J Cancer 2016;138:14-21.

12. Li P, Kaslan M, Lee SH, Yao J, Gao Z. Progress in exosome isolation techniques. Theranostics 2017;7:789.

13. Chiou NT, Ansel KM. Improved exosome isolation by sucrose gradient fractionation of ultracentrifuged crude exosome pellets. Protocol Exchange 2016;10:57.

14. Munagala R, Aqil F, Jeyabalan J, Gupta RC. Bovine milk-derived exosomes for drug delivery. Cancer Lett 2016;371:48-61.

15. Stetefeld J, McKenna SA, Patel TR. Dynamic light scattering: a practical guide and applications in biomedical sciences. Biophys Rev 2016;8:409-27.

16. Bob Carr, Matthew Wright. Nanoparticle tracking analysis, a review of applications and Usage; 2010-12.

17. Hou W, Cronin SB. A review of surface plasmon resonanceenhanced photocatalysis. Adv Functional Materials 2012;23:1612-9.

18. Lobb RJ, Becker M, Wen SW. Optimized exosome isolation protocol for cell culture supernatant and human plasma. J Extracellular Vesicles 2015;4:27031.

19. Andaloussi SE, Mager I, Breakefield XO, Wood MJ. Extracellular vesicles: Biology and emerging therapeutic opportunities. Nat Rev Drug Discovery 2013;12:347-57.

20. Hood RR, Vreeland WN, DeVoe DL. Microfluidic remote loading for rapid single-step liposomal drug preparation. Lab Chip 2014;14:3359-67.

21. Pascucci L, Coccè V, Bonomi A. Paclitaxel is incorporated by mesenchymal stromal cells and released in exosomes that inhibit in vitro tumor growth: A new approach for drug delivery. J Controlled Release 2014;192:262-70.

22. Lee J, Kim J, Jeong M. Liposome-based engineering of cells to package hydrophobic compounds in membrane vesicles for tumor penetration. Nano Lett 2015;15:2938-44.

23. Lee J, Lee $\mathrm{H}$, Goh U. Cellular engineering with membrane fusogenic liposomes to produce functionalized extracellular vesicles. ACS Appl Materials Interfaces 2016;8:6790-5.

24. Akao Y, Nakagawa Y, Hirata I. Role of anti-oncomirs miR-143 and-145 in human colorectal tumors. Cancer Gene Ther 2010;17:398-408

25. Ohno S, Takanashi M, Sudo K. Systemically injected exosomes targeted to EGFR deliver antitumor microRNA to breast cancer cells. Mol Ther 2013;21:185-91.

26. Sun D, Zhuang X, Xiang X. A novel nanoparticle drug delivery system: the anti-inflammatory activity of curcumin is enhanced when encapsulated in exosomes. Mol Ther 2010;18:1606-14.

27. Didiot MC, Hall LM, Coles AH. Exosome-mediated delivery of hydrophobically modified siRNA for huntingtin mRNA silencing. Mol Ther 2016;24:1836-47.

28. Fuhrmann G, Serio A, Mazo M, Nair R, Stevens MM. Active loading into extracellular vesicles significantly improves the cellular uptake and photodynamic effect of porphyrins. J Controlled Release 2015;205:35-44.

29. Haney MJ, Klyachko NL, Zhao Y. Exosomes as drug delivery vehicles for Parkinson's disease therapy. J Controlled Release 2015;207:18-30.

30. Kooijmans SAA, Stremersch S, Braeckmans K. Electroporationinduced siRNA precipitation obscures the efficiency of siRNA loading into extracellular vesicles. J Controlled Release 2013;172:229-38.

31. Kim MS, Haney MJ, Zhao Y. Development of exosomeencapsulated paclitaxel to overcome MDR in cancer cells. Nanomed: Nanotechnol Biol Med 2016;12:655-64.
32. Lamichhane TN, Jeyaram A, Patel DB. Oncogene knockdown via active loading of small RNAs into extracellular vesicles by sonication. Cellular Molecular Bioengineering 2016;9:315-24.

33. Sato YT, Umezaki K, Sawada S. Engineering hybrid exosomes by membrane fusion with liposomes. Sci Rep 2016;6:21933.

34. Podolak I, Galanty A, Sobolewska D. Saponins as cytotoxic agents: a review. Phytochem Rev 2010;9:425-74

35. Simpson RJ, Lim JW, Moritz RL, Mathivanan S. Exosomes proteomic insights and diagnostic potential. Expert Rev Proteomics 2009;6:267-83.

36. Pisitkun T, Johnstone R, Knepper MA. Discovery of urinary biomarkers. Molecular Cellular Proteomics 2006;5:1760-71.

37. Dai S, Zhou X, Wang B. Enhanced induction of dendritic cell maturation and HLA-A*0201-restricted CEA-specific CD8+CTL response by exosomes derived from IL-18 gene-modified CEApositive tumor cells. J Mol Med 2006;84:1067-76.

38. Liu C, Yu S, Zinn K. Murine mammary carcinoma exosomes promote tumor growth by suppression of NK cell function. Immunol 2006;176:1375-85.

39. Salido Guadarrama I, Romero Cordoba S, Peralta Zaragoza O, Hidalgo Miranda A, Rodriguez Dorantes M. MicroRNAs transported by exosomes in body fluids as mediators of intercellular communication in cancer. OncoTargets Ther 2014;7:1327.

40. Mahaweni NM, Kaijen Lambers ME, Dekkers J, Aerts JG, Hegmans JP. Tumour-derived exosomes as antigen delivery carriers in dendritic cell-based immunotherapy for malignant mesothelioma. J Extracellular Vesicles 2013;2:22492.

41. Chaudhuri AA, So AY, Sinha N. MicroRNA-125b potentiates macrophage activation. J Immunol 2011;187:5062-8.

42. Gross JC, Chaudhary V, Bartscherer K, Boutros M. Active wnt proteins are secreted on exosomes. Nat Cell Biol 2012;14:1036-45.

43. Lai RC, Yeo RW, Tan KH, Lim SK. Exosomes for drug delivery-a novel application for the mesenchymal stem cell. Biotechnol Adv 2013;31:543-51.

44. Chaput N. Dendritic cell derived-exosomes: biology and clinical implementations. J Leukocyte Biol 2006;80:471-8.

45. Basu J, Ludlow JW. Exosomes for repair, regeneration and rejuvenation. Expert Opinion Biol Ther 2016;16:489-506.

46. Russo I, Bubacco L, Greggio E. Exosomes-associated neurodegeneration and progression of Parkinson's disease. Am J Neurodegenerative Diseases 2012;1:217.

47. Rajendran L, Honsho M, Zahn TR. Alzheimer's disease $\beta$ amyloid peptides are released in association with exosomes. Proc Natl Acad Sci 2006;103:11172-7.

48. Malm T, Loppi S, Kanninen KM. Exosomes in Alzheimer's disease. Neurochem Int 2016;97:193-9.

49. Costa Silva B, Aiello NM, Ocean AJ. Pancreatic cancer exosomes initiate pre-metastatic niche formation in the liver. Nat Cell Biol 2015;17:816-26.

50. Jarmalaviciutè A, Pivoriunas A. Exosomes as a potential novel therapeutic tool against neurodegenerative diseases. Pharmacol Res 2016;113:816-22.

51. Howitt J, Hill AF. Exosomes in the pathology of neurodegenerative diseases. J Biol Chem 2016;291:26589-97.

52. Lai CPK, Breakefield XO. Role of exosomes/microvesicles in the nervous system and use in emerging therapies. Frontiers Physiol 2012;3:228.

53. Natasha G, Gundogan B, Tan A. Exosomes as immunotherapeutic nanoparticles. Clin Ther 2014;36:820-9.

54. Amoozgar Z, Goldberg MS. Targeting myeloid cells using nanoparticles to improve cancer immunotherapy. Adv Drug Delivery Rev 2015;91:38-51.

55. Hamdy S, Haddadi A, Hung RW, Lavasanifar A. Targeting dendritic cells with nano-particulate PLGA cancer vaccine formulations. Adv Drug Delivery Rev 2011;63:943-55.

56. Luan X, Sansanaphongpricha K, Myers I, Chen H, Yuan H, Sun D. Engineering exosomes as refined biological nanoplatforms for drug delivery. Acta Pharmacol Sin 2017;38:754-63.

57. Qi Y, Xu Q, Wang Y, Yan B, Ren Y, Chen Z. CO2-induced phase engineering: protocol for enhanced photoelectrocatalytic performance of 2D MoS2 nanosheets. ACS Nano 2016;10:2903-9.

58. Morales Kastresana A, Telford B, Musich TA. Labeling extracellular vesicles for nanoscale flow cytometry. Sci Rep 2017;7:1878.

59. Kamerkar S, LeBleu VS, Sugimoto H. Exosomes facilitate therapeutic targeting of oncogenic KRAS in pancreatic cancer. Nature 2017;546:498-503. 
60. Stremersch S, Vandenbroucke RE, Wonterghem EV, Hendrix A, DeSmedt SC, Raemdonck K. Comparing exosome-like vesicles with liposomes for the functional cellular delivery of small RNAs. J Controlled Release 2016;232:51-61.

61. Sun DM, Zhuang XY, Xiang XY, Liu YL, Zhang SY, Liu CR. A novel nanoparticle drug delivery system: the anti-inflammatory activity of curcumin is enhanced when encapsulated in exosomes. Mol Ther 2010;1:606-14.

62. Tian YH, Li SP, Song J, TJ Ji, Zhu MT. A doxorubicin delivery platform using engineered natural membrane vesicle exosomes for targeted tumor therapy. Biomaterials 2014;35:2383-90.

63. Yang TZ, P Martin P, Fogarty B, Brown A. Exosome delivered anticancer drugs across the blood-brain barrier for brain cancer therapy in Danio Rerio. Pharm Res 2015;32:2003-14.
64. MJ Haney, NL Klyachko, YL Zhao, R Gupta, EG Plotnikova, ZJ He. Exosomes as drug delivery vehicles for Parkinsors disease therapy. J Controlled Release 2015;207:18-30.

65. Wahlgren J, Karlson TDL, Brisslert M, Sani FV, Telemo E, Sunnerhagen P. Plasma exosomes can deliver exogenous short interfering RNA to monocytes and lymphocytes. Nucleic Acids Res 2012;40:130.

66. Shtam TA, Kovalev RA, Varfolomeeva EY, Makarov EM. Exosomes are natural carriers of exogenous siRNA to human cells in vitro. Cell Communication Signaling 2013;88:1186-96.

67. Banizs $\mathrm{AB}$, Huang $\mathrm{T}$, Dryden $\mathrm{K}$, Berr SS, Stone JR. In vitro evaluation of endothelial exosomes as carriers for small interfering ribonucleic acid delivery. Int J Nanomed 2014:9:4223-30 